\title{
Hemoglobin Barts to Total Hemoglobin Ratio Measurement
}

National Cancer Institute

\section{Source}

National Cancer Institute. Hemoglobin Barts to Total Hemoglobin Ratio Measurement. NCl Thesaurus. Code C147354.

The determination of the ratio of hemoglobin Barts compared to total hemoglobin present in a sample. The measurement may be expressed as a ratio or percentage. 